\title{
SOBRE LAS REPERCUSIONES AMBIENTALES DE LAS TRANSFORMACIONES DEL PAISAJE: OPORTUNIDADES PARA LA GEOGRAFÍA HISTÓRICA
}

\author{
Pablo Giménez Font \\ Instituto Interuniversitario de Geografía. Universidad de Alicante
}

\section{RESUMEN}

La presente reflexión pretende destacar el valor de los enfoques provenientes de la geografía histórica en el debate internacional sobre las repercusiones ambientales de las transformaciones del paisaje. Centraremos estas ideas en los cambios producidos en los ecosistemas mediterráneos a partir de la reconstrucción de los usos del suelo en los últimos cuatro siglos.

Palabras clave: geografía histórica, paisaje mediterráneo, cambios en los usos del suelo, biodiversidad.

\begin{abstract}
These reflections aim to underline the importance of historical-geographical approaches in the international debate on environmental repercussions of landscape change. We have applied these ideas to the changes produced in Mediterranean ecosystems over the last four hundred years due to land use transformations.
\end{abstract}

Key words: Historical geography, Mediterranean landscape, land use changes, biodiversity.

\section{Enfoque inicial}

La riqueza de los ecosistemas mediterráneos, englobados en los llamados hotspots biodiversity del planeta, se debe a una gran variedad de factores. Las migraciones de especies vegetales a lo largo de la evolución biogeográfica, las condiciones climáticas particulares, la diversidad litológica, la disposición estructural del relieve o la configuración de su red hidrográfica explican la gran diversidad de especies - singularmente vegetales - , muchas de las cuales son endémicas. Pero este hecho también es debido la intensa y plurisecular humanización de sus riberas, en forma de grandes y complejos agrosistemas. De hecho, en 
las tierras circunmediterráneas, la fitodiversidad no es sinónimo de formaciones cercanas al óptimo. En el caso concreto de la endemoflora valenciana, como señala Marco (2007), resulta llamativo que el hábitat que aporta el menor porcentaje de endemismos es el de los bosques mixtos de quercíneas y formaciones caducifolias (en torno al 3\%), mientras que formaciones subarbustivas que lo sustituyen (entre otros, tomillares o salviares), relacionadas con los procesos de deforestación y manejo plurisecular del territorio, pueden alcanzar hasta el $50 \%$ del total.

Por tanto, los ecosistemas de larga coevolución del Mediterráneo tienen en su devenir histórico parte de su esencia, que puede explicarse desde multitud de puntos de vista. La conjunción de enfoques en torno a estas cuestiones, lejos de significar un desencuentro entre disciplinas, está resultando un acontecimiento científico muy prolífico y relevante en la Ciencia actual. Se trata de una respuesta a los complejos y masivos cambios globales que se están sucediendo, de forma creciente, en el último siglo. Y es así, en parte, por la presencia de un concepto de integración como es el Paisaje.

Objeto fundacional de la Geografía moderna, hoy existen multitud de definiciones para este concepto polisémico, cuyo análisis se ha posicionado, especialmente en las dos últimas décadas, como una válida propuesta de interpretación bajo narrativas diversas, mucho más conectadas entre sí de lo que se hace ver.

La toma de conciencia sobre la masiva transformación, más o menos irreversible, de un bien común que concierne aspectos territoriales como el patrimonio natural y cultural o conceptos difícilmente objetivables como la calidad de vida y el bienestar social, ha propiciado recientemente la toma de posiciones en defensa del paisaje. En Europa, dicha inquietud se vio materializada en la firma del Convenio Europeo del Paisaje (CEP) (Consejo de Europa, Florencia, 2000), el primer tratado internacional dedicado específicamente a la salvaguarda y gestión de los paisajes (Mata, 2006), que en España fue ratificado en 2008. Dicho convenio ha multiplicado las políticas de paisaje a escalas comunitarias, nacionales y regionales (Paül y Queralt, 2009) y ha incentivado una prolífica línea de investigación, de carácter multidisciplinar, en torno a la evolución, la percepción, la protección y la gestión de los paisajes.

El CEP (cap. 1, art.1a) define paisaje como «cualquier parte del territorio, tal y como la percibe la población, cuyo carácter sea el resultado de la acción y la interacción de factores naturales y/o humanos». Una definición genérica, pero abierta a multitud de lecturas relacionadas con el territorio, la percepción y a la relación entre naturaleza y sociedad. En primer lugar, cabe señalar que la importancia del elemento perceptivo en el análisis del paisaje puede resultar problemática, más aún en un contexto social cada vez más urbanita y desvinculado del mundo rural, que puede no asumir la complejidad que existe en un paisaje claramente marcado por la actividad humana. Este hecho ha determinado una serie de problemas derivados de una gestión basada en los que algunos autores han denominado «ambietalismo ideológico» (Ojeda, 1999) mal entendido.

La definición del CEP también se refiere a la vinculación entre naturaleza y sociedad, acepción especialmente sugerente desde nuestro punto de vista porque indica cambio, evolución, dinamismo y, en definitiva, complejidad. De esta forma, el paisaje encuentra en el elemento histórico una de sus bases analíticas, ya que el conocimiento preciso de su evolución aporta los elementos para el conocimiento del presente y para la adopción de medidas de gestión eficiente. La percepción del mismo incluye, igualmente, una serie de características visuales que derivan de factores sociales y ambientales que, con distinto rango y de forma más o menos reciente, general o específica, derivan del distinto control y uso de los recursos naturales a lo largo de la historia.

Dado que, en el Mediterráneo, parte de la biodiversidad se relaciona directamente de estos usos agrosilvopastoriles, es necesario comprender la dinámica histórica de estos pai- 
sajes para explicar la riqueza del presente, los procesos de cambio a lo largo del tiempo e intuir la evolución futura. Esta tarea involucra e integra a multitud de disciplinas del campo de las ciencias sociales y naturales, por lo que se trata de una oportunidad para la Geografía debido a su carácter de ciencia de frontera, a sus lenguajes amplios y a su capacidad integradora y sintética. La emergencia de este tema en la última década queda reflejada en la numerosa bibliografía al respecto (Swetnam et al. 1999; Iriarte y Sabio, eds. 2003; Pèlach, 2006; Girel, 2006; Jacob-Rousseau, 2009; Nadal et al. 2009). Aspecto que determina la existencia de enfoques diversos para caracterizar la dinámica de los paisajes donde los geógrafos han desarrollado su investigación. Sintéticamente podemos diferenciar dos grandes conjuntos de estudios a partir de las técnicas utilizadas y la escala temporal:

- estudios centrados en cambios producidos en los últimos 10.000 años (Holoceno), donde pesan especialmente los factores vinculados al cambio climático y que, por tanto, utilizan «archivos naturales» a partir de técnicas diversas como los análisis palinológicos, antracológicos, sedimentológicos, etc.

- y otros de carácter histórico, con escalas temporales más recientes, que utiliza fuentes diferentes, básicamente documentales de diversa procedencia y tipología.

Entre la diversidad de enfoques encontramos los planteamientos vinculados a la Ecología del Paisaje, una línea convergente con la Geografía (Marty, et al., 2006) que tiene uno de sus precedentes originales en los planteamientos de Carl Troll (Terradas, 2003). También los estudios de carácter socioecológico, ligados a la cuestión del «metabolismo social», que predominan en la reciente historia rural española y europea (Garrabou y Naredo, coords. 2008; Tello, coord. 2006; Agnoletti, 2007). Enfoques con una gran proyección actual, que confluyen en el paisaje por su capacidad de expresar los cambios en los usos y coberturas del suelo.

No obstante, en muchas ocasiones unos y otros se centran excesivamente en procesos ecológicos, por un lado, y en procesos de raíz socioecológica, por el otro. El enfoque geográfico, de larga tradición, puede aportar una mirada territorial más completa, al introducir la dimensión cultural, perceptiva y subjetiva del paisaje, especialmente válida para la vertiente aplicada de esta ciencia: la ordenación y gestión del territorio, que ha renovado el interés por el paisaje (Antrop, 2005). La clásica corología del paisaje, la descripción y caracterización de la morfología del mosaico territorial del método geográfico tradicional, ha sido ampliamente mejorada por diversas líneas de trabajo que inciden en el factor temporal y en los sistemas que organizan las estructuras espaciales en su interpretación. El sistema GTP de Bertrand (2006), los planteamientos de García Fernández (1978) o los trabajos de Martínez de Pisón (1998), por citar algunos clásicos recientes, representan las bases de la renovación conceptual de los estudios geográficos de paisaje en España.

En definitiva, lo que algunos autores han denominado la línea dinámica o funcional y evolutiva del paisaje (Sanz Herráiz, 2008: 454) ha constituido un ámbito multidisciplinar que, en nuestra opinión, resulta un compendio general y un lugar de encuentro de los puntos de vista referidos. Se habla así de Arqueología del Paisaje (Leveau, 2000; Bolós, 2004) o, directamente, de Historia del Paisaje, que para muchos es sinónimo o parte fundamental de la historia ambiental (Sieferle, 2001; Iriarte y Sabio, 2003).

\section{Hacia una geografía de los procesos}

Resulta cuanto menos llamativo que Baker (2007), uno de los principales autores que han reflexionado en torno a la Geografía histórica, haya centrado parte de sus argumentos en si se puede hablar de «geografía histórica» como tal, dada su ambigüedad epistemológica. Ciertamente, como afirma este geógrafo, en las últimas décadas la historiografía se ha acer- 
cado al estudio de las relaciones recíprocas entre la sociedad y la naturaleza - incluyendo aquí el paisaje - más como «historiadores del medio ambiente» que como geógrafos. Tal sería el caso de obras de impacto como las de McNeill (2003) o Grove y Rackham (2001). No obstante, una característica generalmente compartida es que los distintos modos de abordar la dinámica del paisaje se centran en los procesos involucrados en el mismo. Se trata de una opción, la del análisis de procesos, igualmente abierta y polisémica, pero que bebe de una larga tradición de estudios de Geografía histórica y es una de las corrientes más desarrolladas en la Geografía actual (Aspinall, 2010).

Con ocasión de esta apreciación, cabe destacar dos aspectos básicos que pueden considerarse determinantes y diferenciadores de los distintos tipos de estudios de la evolución del paisaje. En primer lugar, la escala de análisis temporal, vinculada a las fuentes de documentación. Sin contar con los estudios de largo recorrido temporal (miles de años) numerosos trabajos presentan un punto de partida generalizado en el ecuador del siglo XX. En España parten de la extraordinaria calidad y amplitud de la información aportada por la fotografía aérea de 1956 y es común en los trabajos de ecología del paisaje y geografía. Sin embargo, a pesar de la entidad de los cambios acontecidos en estos últimos 50 años, sabemos que las dinámicas naturales tienen una dimensión temporal mucho más amplia. La historia rural, por el contrario, presenta periodos temporales más prolongados - ss. XVI al XIX, principalmente- y, aunque el detalle aportado por la documentación de archivo normalmente no es equiparable a la anterior, en algunos espacios se han logrado niveles de detalle admirables, tal y como se demuestra en el modélico estudio sobre la Albufera de Valencia de Sanchis Ibor (2001).

Por otro lado, está el sentido de la interpretación. El planteamiento general de numerosos trabajos de Geografía histórica se centra, fundamentalmente, en los aspectos perceptibles del paisaje, es decir, en la materialidad de sus formas o en los usos y coberturas del suelo a lo largo de una diacronía concreta. Sin embargo, siguiendo las definiciones de González Bernáldez (1981: 3), no se pretende únicamente reconstruir un fenosistema, entendido como un «conjunto de componentes perceptibles en forma de panorama, escena o paisaje»; sino que se pretende profundizar en el criptosistema, ese «complemento de más difícil observación, que proporciona la explicación que falta para la comprensión del geosistema». En cierta forma, la interpretación de paisajes reconstruidos para distintas épocas, puede afrontarse a partir de su consideración como parte visible de un ecosistema, de forma que los cambios perceptibles serían, a su vez, la consecuencia de las transformaciones ocurridas en la estructura y funcionamiento de cada ecosistema. En demasiadas ocasiones ha prevalecido la primera acepción, la referida a las formas y percepciones del paisaje, cuando es precisamente la determinación de las causas que lo explican una oportunidad para la perspectiva geográfica. La complejidad del paisaje requiere, más allá de una visión social, ecológica o socioecológica, una perspectiva a nivel de ecosistema.

\section{Los valores ecológicos del paisaje y la Geografía histórica ambiental}

Estas breves referencias pretenden significar cómo la complejidad de miradas diluyen herramientas metodológicas y conceptuales que, de forma cada vez más generalizada, empiezan a intercambiarse y combinarse (Vila et al. 2006; Gurrutxaga y Lozano, 2008). De hecho las conclusiones de estudios realizados bajo perspectivas diferentes vienen a ser, de una u otra forma, convergentes y hacen referencia a los problemas derivados de los intensos cambios paisajísticos sufridos en el último siglo y, especialmente, en las últimas seis décadas. No obstante, la reconstrucción del paisaje mediterráneo en los últimos siglos es una línea de investigación creciente (Harfouche, 2005), con los ejemplos destacables de 
Liguria (Gentili et al. 2009), Lesvos (Kizos y Kouloiri, 2006) o la Toscana (Agnoletti, 2007), entre otros. En España resulta especialmente sugerente la línea desarrollada en los Pirineos (Briffaud, 1994; Utrillas et al. 2003; Matamala et al.2005, entre otros). Todos ellos realizan sus estudios a partir de diversas fuentes documentales y aportan una marcada perspectiva multidisciplinar. No cabe duda, pues, que aquí reside una de las propuestas más sólidas y rentables para analizar la complejidad del paisaje. Y cabe preguntarse, en este contexto amplio y difuso de enfoques, sobre el papel que puede desempeñar la Geografía histórica.

El paisaje mediterráneo, entendido como un paisaje plenamente (poli)cultural, posee unos valores ecológicos estrechamente vinculados al aprovechamiento humano. A partir de la base de unos condicionamientos físicos determinados (geomorfológicos, climáticos, edáficos y bióticos), los paisajes rurales se han caracterizado por una marcada heterogeneidad y complejidad de su estructura (setos, márgenes de piedra, bosques galería, árboles aislados, policultivo, regadío, pastizales, áreas de monte y bosque, vías pecuarias, etc.), fruto de una organización determinada del espacio, caracterizada mayoritariamente por una elevada eficiencia en el uso de la energía y los nutrientes (De Miguel, 1999). El mosaico heterogéneo de usos tradicionales del territorio y su funcionalidad es la base de la riqueza ecológica, debido a que diversos ecosistemas se conectan, se combinan y se complementan a lo largo del tiempo. Por tanto, conocer la distribución espacial de los usos del territorio puede ofrecer información sobre procesos ecológicos como el ciclo de los nutrientes, la dispersión de las plantas y animales o las interacciones entre unos y otros, bases de la moderna Ecología del Paisaje (Turner, 2005).

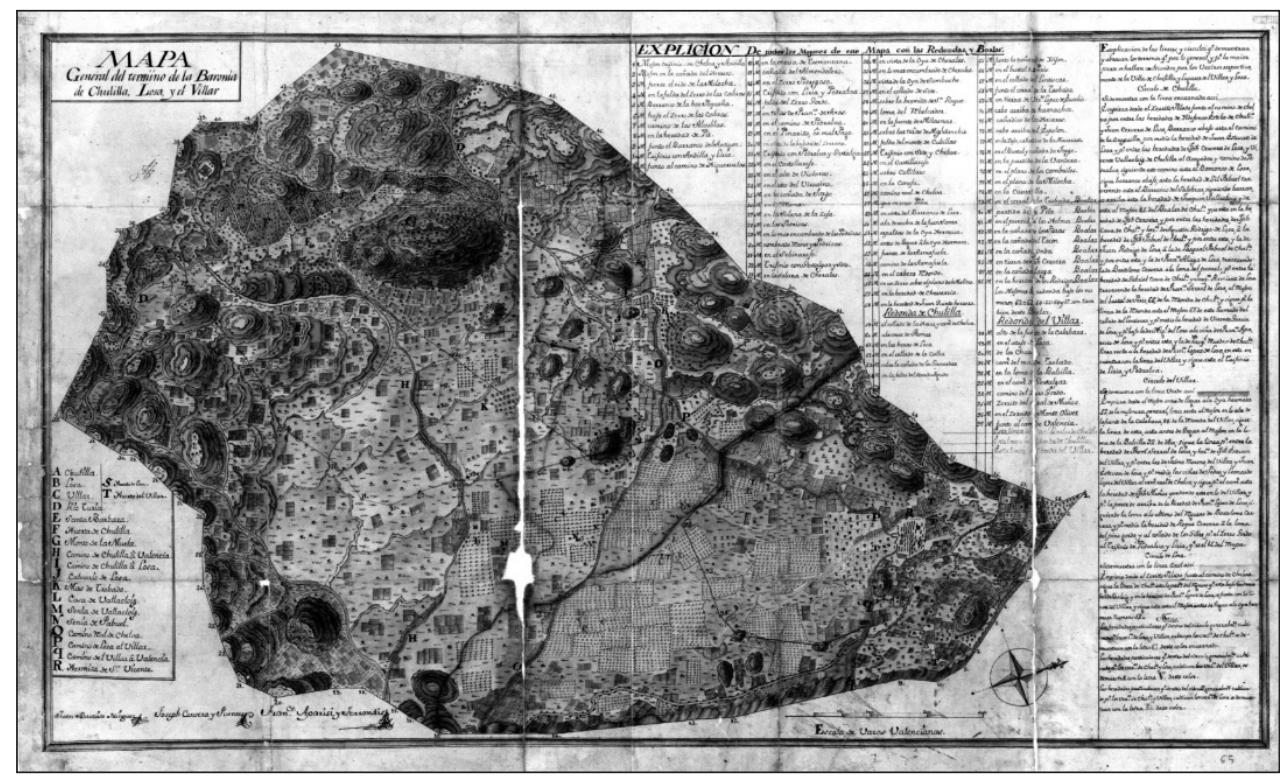

FiguRa 1. Mapa General del término de la baronía de Chulilla, Losa y Villar (Valencia, 1770). Ejemplo de una valiosa cartografía que representa una gran diversidad de usos del suelo, vinculados con tipos de cultivo y de vegetación natural, concentrada principalmente en los bovalares aún activos. Mapas como éste, junto con la documentación escrita que los acompañan, pueden ser el punto de partida de estudios de dinámica del paisaje. Arxiu del Regne de València, M y P. $\mathrm{n}^{\circ} 224$. 
En la segunda mitad del siglo XX se han producido cambios extraordinarios en la distribución y características de los usos del suelo: se han modificado las coberturas, al desaparecer las formas de policultivo o introducir nuevas plantaciones, al deforestar o reforestar; se han transformado las estructuras paisajísticas - algunas de ellas milenarias - como la red caminera o el parcelario; o directamente se ha urbanizado masivamente, comportando cambios radicales derivados del sellado de suelos. Todos ellos, pueden entenderse como cambios morfológicos que han ocasionado impactos profundos de tipo geomorfológico, edáfico, biológico, cultural y estético, que han centrado el interés de las diversas disciplinas referidas líneas atrás. No obstante, el desarrollo de esta línea de investigación abre una serie de amplias cuestiones que entendemos como una oportunidad para la Geografía:

- La necesidad de escalas temporales más prolongadas para el análisis de los cambios del paisaje (a través del manejo de fuentes diversas)

- La determinación de los procesos motivadores de dichos cambios (desde un punto de vista amplio e integral, en el contacto entre la Geografía y la Historia)

- El análisis, la representación y la síntesis cartográfica

La reconstrucción de la dinámica del paisaje en los últimos siglos puede arrojar información sobre procesos actuales que derivan de cambios ocurridos más allá de la segunda mitad del siglo XX. Permite ampliar perspectivas sobre cambios territoriales y ambientales que requieren largos períodos de tiempo y valorar y comparar la capacidad de respuesta y adaptación de distintos sistemas naturales y a distintas escalas.

\section{Sobre la reconstrucción de paisajes de referencia}

La reconstrucción de paisajes históricos, por tanto, se puede afrontar desde diferentes escalas temporales y espaciales. La decisión deriva de la perspectiva inicial, de las fuentes de información disponibles y de los objetivos perseguidos. En todo caso, consiste en reconstruir, con mayor o menor detalle, los usos y sus coberturas de un mismo espacio en determinadas épocas. Una reconstrucción que no deja de ser estática pero que, en conjunto, proporciona una evolución dinámica de los cambios acontecidos a lo largo de un período temporal determinado. Se trata de un primer paso necesario para interpretaciones más profundas, a nivel de ecosistema, que pueden proporcionar información muy valiosa. Por ejemplo, centrando el interés en ecotonos, la interpretación de estos paisajes de referencia a partir de documentación histórica se ha erigido como un método eficaz para definir estrategias de conservación y restauración de ecosistemas (Fritschle, 2008; Foster, 2002; Etter et al. 2008).

Como ejemplo de conexión o relación entre hábitats singulares y procesos históricos, el estudio de los bosques de ribera presenta aspectos muy sugerentes que están siendo estudiados desde hace décadas (Panareda, 2009). Los márgenes de los ríos fueron, en muchos casos, espacios comunales donde se obtenía leña y pastos, regidos por usos consuetudinarios. Las fuentes documentales y cartográficas ofrecen, especialmente para los principales cursos fluviales, información muy valiosa, ya que históricamente las formaciones de ribera fueron mucho más continuas, diversas y abundantes que en la actualidad. Con el desmantelamiento del Antiguo Régimen, se aceleró el proceso de enajenación de numerosos espacios inmediatos al río, con los consiguientes cambios de uso, que pueden resumirse en el paso de mosaicos complejos de formaciones ripícolas a campos de cultivos, con la intensificación añadida del aprovechamiento poco sostenible de los recursos forestales (figura 2). En multitud de cursos fluviales, las consecuencias sociales y ambientales pasaron por la desaparición de antiguos equilibrios entre la comunidad y su entorno, con consecuencias sobre la flora y fauna específica y la dinámica de los propios sistemas fluviales (Mateu, 


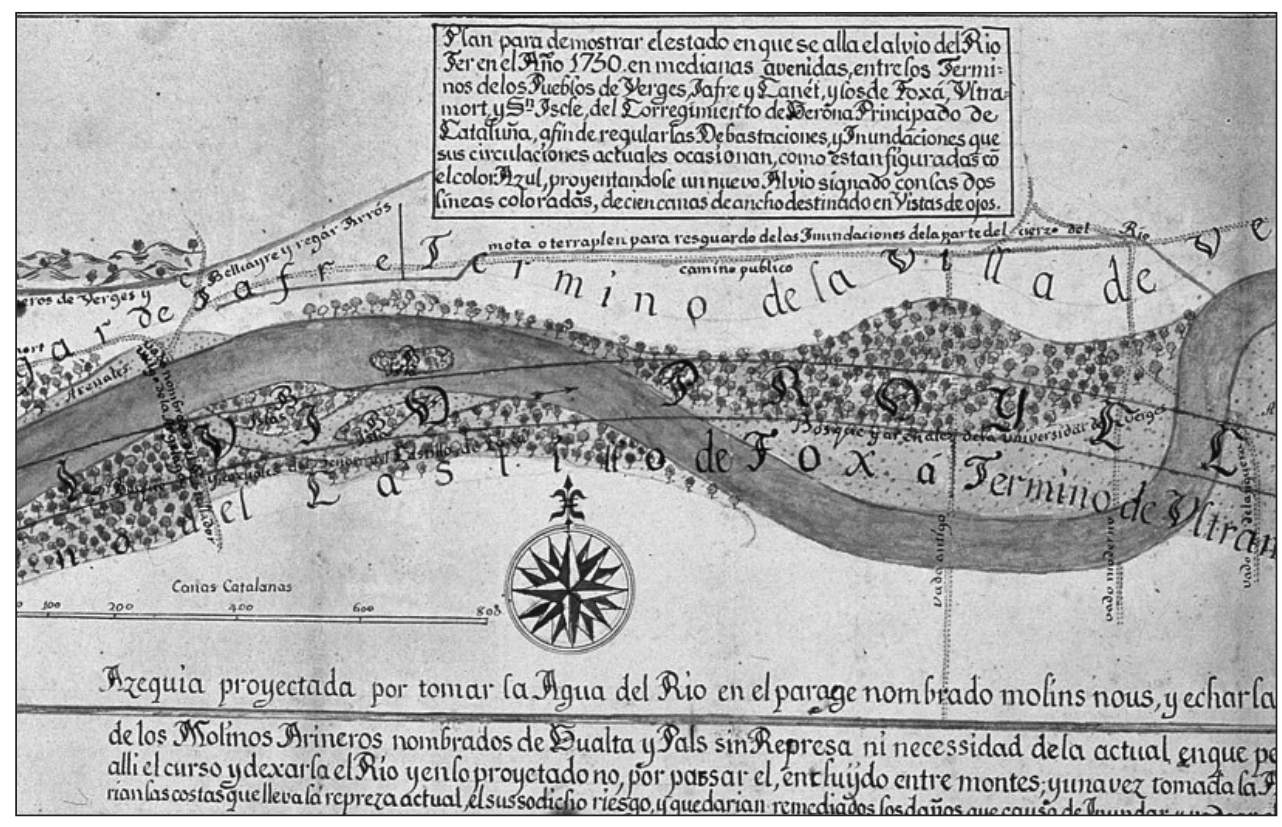

Figura 2. Fragmento del plano de Bernardo Ferrer i Mir (1750) sobre el Riu Ter a la altura de Verges. Se representa los bosques de ribera hoy desaparecidos, algunos de los cuales, como el de la imagen (bosques y arenales de la universidad de Verges), eran de propiedad comunal. Fuente: Archivo de la Corona de Aragón, $M y P$ - 550 .

2007). De igual forma, la modificación de las dinámicas fluviales — mucho más intensa a partir del siglo XIX con el desarrollo de grandes infraestructuras hidráulicas - ha alterado significativamente la composición de la vegetación de ribera.

Lógicamente, el paisaje vegetal - como resultado de las interacciones abióticas y bióticas que forman el complejo ecológico - ha sido el que mayor atención ha recibido. De hecho, existe una prolífica línea de investigación sobre las formaciones vegetales históricas, que se ha desarrollado especialmente en América donde el proceso de colonización europea iniciada en el siglo XVI permite partir de esquemas más o menos climácicos, aunque recientes investigaciones descartan generalizar esta idea (Butzer, 1993; Etter et al. 2008; Sanderson, 2009). En el mundo Mediterráneo, sin embargo, la complejidad es mucho mayor debido a la intensa humanización y a la propia configuración física de las riberas de este mar, un amplio conjunto de ecosistemas de transición y frágil equilibrio (Blondel y Aronson, 1999; Horden y Purcell, 2000). En este espacio, precisamente, la diversidad material y simbólica de los paisajes ha dotado de gran importancia a la escala local (Cevasco, 2008). Esta idea entronca con las metodologías empleadas en la (no tan) «nueva historia rural», que desde hace décadas focaliza una investigación en un estudio de caso que, a modo de subsistema, es asimilado en gran medida como un experimento de laboratorio. Los resultados obtenidos se contextualizan en un orden superior, pero a su vez pueden redefinir ese orden preestablecido con aportaciones novedosas que pueden ofrecer luz sobre la compleja red de interacciones entre una comunidad y su medio ambiente, posiblemente inapreciables a escalas más generales. El creciente número de publicaciones - entre las que destacaría los trabajos del Laboratorio di Archaeologia e Storia Ambientale de la Universidad de Génova 
o la larga experiencia científica de Butzer (2005) en el Mediterráneo- resaltan la importancia del análisis de la trayectoria histórica de las comunidades rurales en la comprensión de los ecosistemas actuales.

Resultados como los anteriores señalan, en definitiva, cómo la perspectiva histórica permite enriquecer el conjunto de relaciones complejas que interactúan en el paisaje, permitiendo valorar las lecciones del pasado, las posibles tendencias de cambio en el futuro para enfocar, finalmente, las discusiones y aspectos más relevantes para la investigación y la gestión eficiente de paisajes. Cualidad que se afronta como un reto para las políticas conservacionistas y gestores ambientales que han de valorar las herencias que todo espacio acumula, fruto de procesos de cambio natural y de la actividad humana. Ante este planteamiento, surgen cuestiones relacionadas con cómo valorar y cómo conservar paisajes que de por sí son dinámicos o que basan su particularidad en formas de organización humana que actualmente se encuentran en un proceso irreversible de desaparición (Foster, 2002). Buena muestra de ello es que muchos de los actuales espacios protegidos en España contienen extensas superficies de espacios agrarios o responden directamente a la presencia de esta actividad, como es el caso de las dehesas (Pinea y Montalvo, 1995), de forma que se plantea la necesidad de conservar esas actividades tradicionales, más que conservar los escasos espacios con una baja antropización. Éste es uno de los principales retos para la conservación y gestión de la biodiversidad en España.

\section{A modo de conclusión}

Muchas de las características de los ecosistemas (formaciones vegetales, propiedades del suelo y fauna) se explican analizando sus trayectorias a largo plazo. Pero aunque las fuentes sedimentarias se consideran los archivos reales de la historia ambiental de los paisajes culturales (Moreno y Montanari, 2008: 35), frecuentemente no es necesario llegar hasta dataciones tan distantes - donde hay una reducción evidente de fuentes documentales - para comprobar cómo algunos acontecimientos del pasado reciente son la clave para explicar la dinámica del paisaje actual (Padilla, 1995; Marco, 2001 y 2010; Foster et al. 2003), lo cual representa una oportunidad para la Geografía histórica de corte ambiental. De esta forma, los usos del suelo y las herencias de las transformaciones tienen una importancia evidente en todos los ecosistemas y proporcionan uno de los motivos fundamentales por los que la investigación histórica sigue siendo un enfoque básico en los estudios de biogeografía y ecología y en los procesos de planificación para la conservación (Agnoletti, ed. 2006).

Retomando las ideas de partida, la reconstrucción de los ambientes pasados forma parte de una de las más antiguas tradiciones de los trabajos de Geografía histórica, lo que también incluye las percepciones y actitudes culturales en torno a la naturaleza. Como ciencia antropocéntrica, la Geografía incide en la compleja cuestión de la influencia del hombre en el cambio ambiental, un gran estimulante y focalizador de numerosas contribuciones de distinto signo, y entre su diversidad de prácticas destaca el estudio diacrónico o retrospectivo del paisaje, tal vez, nuestra principal aportación a la historia ambiental.

Los tradicionales trabajos regionales de tipo más bien descriptivo, que caracterizaban los elementos del paisaje como evidencia científica, pueden replantearse ahora bajo estos nuevos enfoques. A pesar de la tradición en Geografía histórica, la labor de reconstruir paisajes como forma de medir el impacto de las actividades humanas sobre los ecosistemas, resulta muy dificultosa y requiere de técnicas diversas. Las construcciones suelen ser estáticas, y desde un planteamiento regresivo se puede establecer de forma más o menos detallada su dinámica, intentando recomponer el grado de transformación del paisaje en un momento determinado de la historia. Para ello, generalmente ya son necesarias técnicas muy espe- 
cíficas y la participación de otros expertos como arqueólogos, historiadores, economistas, botánicos o ecólogos. La información obtenida desde una amplia gama de fuentes diversas puede permitir inferir cambios ambientales ocasionados por las actividades antropogénicas o identificar tendencias de cambio a partir de la interpretación de las respuestas humanas a las mismas. Dependiendo de los datos y de los análisis empleados, dichas tendencias se podrán deducir con una fiabilidad aceptable; por el contrario, la explicación de los posibles cambios ecológicos siempre resultará mucho más compleja y abierta al debate.

\section{Agradecimientos}

Este trabajo se ha elaborado en el marco del Proyecto de Investigación Las unidades básicas de paisaje agrario de España: identificación, delimitación, caracterización y valoración (CSO2009-12225-C05-03), financiado por el Ministerio de Ciencia e Innovación.

\section{Bibliografía}

AGNOLETTI, M. (ed.) (2006): The Conservation of Cultural Landscapes, CAB International, Wallingford y New York.

AGNOLETTI, M. (2007): «The degradation of traditional landscape in a mountain area of Tuscany during the 19th and 20th centuries: Implications for biodiversity and sustainable management», Forest Ecology and Management, $\mathrm{n}^{\circ}$ 249, pp. 5-17.

ANTROP, M. (2005): «Why landscapes of the past are important for the future», Landscape and Urban Planning, $\mathrm{n}^{\mathrm{o}}$ 70, pp. 21-34.

ASPINALL, R. (2010): «A Century of Physical Geography Research in the Annals», Annals of the Association of American Geographers, $\mathrm{n}^{\circ}$ 100(5), pp. 1049-1059.

BAKER, A.R.H. (2007): «Classifying geographical history» The Professional Geographer, $\mathrm{n}^{\circ} 59$ (3), pp. 344-356.

BLONDEL, J. y ARONSON, J. (1999): Biology and Wildlife of the Mediterranean Region, University Press, Oxford, 350 pp.

BOLÓS, J. (2004): Els orígens medievals del paisatge català. Publicacions de l'Abadia de Montserrat, Barcelona, $463 \mathrm{pp}$.

BRIFFAUD, S. (1994): Naissance d'un paysage. La montagne pyrénéenne à la croisée des regards, $X V I^{\circ}-X I X^{\circ}$ siècle, Université de Toulouse II, Toulouse et Tarbes, $622 \mathrm{pp}$.

BUTZER, K.W. (1993): «No eden in the New World», Nature, n 362, pp. 15-17.

BUTZER, K. W (2005): «Enviromental history in the Mediterranean world: cross-disciplinay investigation of cause-and-effect for degradation and soil erosion», Journal Archaeological Science, $\mathrm{n}^{\circ} 32$, pp. 1.773-1.800.

CEVASCO, R. (2007): Memoria verde. Nuovi spazi per la geografia, Diabasis, Reggio Emilia, 318 pp.

CONSEJO DE EUROPA (2000): Convenio Europeo del Paisaje. Florencia, 20 de octubre de 2000. (Fuente: Ministerio de Medio Ambiente, Rural y Marino: http://www.mma. es/portal/secciones/desarrollo_territorial/paisaje_dt/convenio_paisaje/

DE MIGUEL, J.M. (1999): «Naturaleza y configuración del paisaje agrosilvopastoral en la conservación de la diversidad biológica en España», Revista Chilena de Historia Natural, $\mathrm{n}^{\circ}$ 72, pp. 547-557.

ETTER, A.; McALPINE, C. y POSSINGHAM, H. (2008): «Historical Patterns and Drivers of Landscape Change in Colombia Since 1500: A Regionalized Spatial Approach», Annals of the Association of American Geographers, no 98(1), pp. 2-23. 
FOSTER, D.R. (2002): «Insights from historical geography to ecology and conservation: lessons from the New England landscape», Journal of Biogeography, ${ }^{\circ} 29$, pp. 1.569-1.575.

FOSTER D.R.; SWANSON, F.; ABER, J.; BURKE, I.; BROKAW, N.; TILMAN, D. y KNAPP, A. (2003): «The Importance of Land-Use Legacies to Ecology and Conservation», Bioscience, $\mathrm{n}^{\circ}$ 53-1, pp. 77-88.

FRITSCHLE, J.A. (2008): «Reconstructing historic ecotones using the public land survey: the lost prairies of Redwood National Park», Annals of the Association of American Geographers, no 98(1), pp. 24-39.

GARCÍA FERNÁNDEZ, J. (1975): Organización del espacio y economía rural en la España Atlántica. Siglo XXI, Madrid, 332 pp.

GARRABOU, R. y NAREDO, J.M. (eds.) (2008): El paisaje en perspectiva histórica: formación y transformación del paisaje en el mundo mediterráneo, Institución «Fernando el Católico», Zaragoza, 414 pp.

GENTILI, R.; GENTILI, E. y SGORBATI, S. (2009): «Crop changes from the XVI century to the present in a hill/mountain area of Eastern Liguria (Italy)», Journal of Ethnobiology and Ethnomedicine, $\mathrm{n}^{\circ}$ 5(9), pp. 1-12.

GIREL, J. (2006): «Quand le passé éclaire le present: écologie et histoire du paysage» Géocarreofour, $\mathrm{n}^{\mathrm{o}}$ 81(4), pp. 249-264.

GONZÁLEZ BERNÁLDEZ, F. (1981): Ecología y paisaje. Blume, Madrid, 250 pp.

GROVE, A.T. y RACKHAM, O. (2001): The Nature of Mediterranean Europe. An Ecological History. Yale University Press, New Haven, 384 pp.

GURRUTXAGA, M. y LOZANO, P. (2008): «Ecologia del paisaje. Un marco para el estudio integrado de la dinámica territorial y su incidencia en la vida silvestre», Estudios Geográficos, $\mathrm{n}^{\circ}$ 265, pp. 519-543.

HARFOUCHE, R. (2005): «Retenir et cultiver le sol sur la longue durée: les terrasses de culture et la place du bétail dans la montagne méditerranéenne», Antrophozoologica, $\mathrm{n}^{\mathrm{o}}$ 40(1), pp. 45-80.

HORDEN, P. y PURCELL, N. (2000): The Corrupting Sea. A study of Mediterranean history. Blackwell Publishing, Oxford, $761 \mathrm{pp}$.

IRIARTE, I. y SABIO, A. (2003): «Historia del Paisaje e Historia Ambiental» en La construcción histórica del paisaje agrario en España y Cuba. Los Libros de la Catarata, Madrid, pp. 9-24.

IRIARTE, I. y SABIO, A. (eds.) (2003): La construcción histórica del paisaje agrario en España y Cuba. Los Libros de la Catarata, Madrid, 285 pp.

JACOB-ROUSSEAU, N. (2009): «Géohistoire/Géo-histoire: quelles methods pour quell récit» Géocarrefour, n ${ }^{\circ}$ 84(4), pp. 211-216.

KIZOS, T. y KOULOURI, M. (2006): «Agricultural landscape dynamics in the Mediterranean: Lesvos (Greece) case study using evidence from the last three centuries», Environmental Science \& Policy, $\mathrm{n}^{\circ}$ 9, pp. 330-342.

LEVEAU, Ph. (2000): «Les paysages aux époques historiques: un document archéologique», Annales. Historie, Sciences Sociales, ${ }^{\circ}$ 55(3), pp. 555-582.

MARCO MOLINA, J.A. (2001): Aitana como espacio singular. Perspectiva geográfica de una montaña mediterránea. Universidad de Alicante, Alicante, $107 \mathrm{pp}$.

MARCO MOLINA, J.A. (2007): «Transformaciones y alteraciones de los paisajes vegetales: de la Amazonía a la cuenca del Mediterráneo». En: Alteración y Regeneración de Espacios Naturales. Universitat de València, València, pp. 59-77.

MARCO MOLINA, J.A. (2010): El esparto y los atochares: una aproximación a su significado, aprovechamiento e impronta en el paisaje, Ajuntament-Cercle d'Estudis Sequet però Sanet, San Vicent del Raspeig, 66 pp. 
MARTÍNEZ DE PISÓN, E. (1998): «El concepto de paisaje como instrumento de conocimiento ambiental» en Paisaje y Medio Ambiente, Fundación Duques de SoriaUniversidad de Valladolid, Valladolid, pp. 9-28.

MARTY, P.; LEPART, J.; CAPLAT, P. (2006): «Géographie et écologie des paysages: Quelles relations ?» Bulletin de l'Association de Géographes Français, n 3, pp. 355-367.

MATA, R. (2006): «Un concepto de paisaje para la gestión sostenible del territorio» en Mata, R. y Tarroja, A. (eds): El paisaje y la gestión del territorio. Criterios paisajísticos en la ordenación del territorio y el urbanismo. Diputació de Barcelona-UIMP, Barcelona, pp. 17-40

MATAMALA, N.; PÈLACHS, A.; SORIANO, J.M. (2006): «La utilidad del catastro como fuente para estudios de biogeografía cultural» en III Congreso Español de Biogeografía, Universidad del País Vasco, Bilbao, pp. 126-133.

MATEU, J.F. (2007): «Público y privado en los espacios naturales españoles» en Espacios públicos-espacios privados. Un debate sobre el territorio, Asociación de Geógrafos Españoles, Santander, pp. 209-223.

MORENO, D. y MONTANARI, C. (2008): «Beyond perception: Towards a historical ecology of rural landscape in Italy», Cuadernos Geográficos, n 43, pp. 29-50.

NADAL, J.; PÈLACHS, A.; SORIANO, J.M.; MOLINA, D.; CUNILL, R.; BAL, M.C. (2009): «Mètodes per al estudi transdisciplinari del paisatge d'àrees de muntanya», Documents d'Anàlisi Geogràfica, no 55 , pp. 147-179.

OJEDA, F. (1999): «Naturaleza y desarrollo: cambios en la consideración política de lo ambiental durante la segunda mitad del siglo XX», Papeles de Geografía, no 30 , pp. 103-118.

PADILLA, M.A. (1994): El poblamiento vegetal en las sierras de Peñarroya y Cuartel: aspectos evolutivos y situación actual, Instituto de Cultura Juan Gil-Albert-Universidad de Alicante, Alicante, $107 \mathrm{pp}$.

PANAREDA, J.M. (2009): «Evolución en la percepción del paisaje de ribera», Boletín de la Asociación de Geógrafos Españoles, n 51, pp. 305-320.

PAÜL, V. y QUERALT, A. (2009): «Les politiques i els instruments de protección, gestió i ordenació del paisatge a l'Estat español» en Ordenació i gestió del paisatge a Europa, Observatori del Paisatge de Catalunya, Olot, pp. 64-103.

PÈLACHS, A. (2006): «Algunes reflexions sobre geografía, paisatge i geohistòria ambiental» Documents d'Anàlisi Geogràfica, no 48, pp.179-192.

SANCHIS IBOR, C. (2001): Regadiu i canvi ambiental a l'Albufera de València. València, Universitat de València, $332 \mathrm{pp}$.

SANDERSON, E. W. (2009): Mannahatta. A Natural History of New York City. Abrams, Nueva York, 352 pp.

SANZ HERRÁIZ, C. (2008): «Los científicos de la Tierra y la evolución de los estudios sobre el paisaje en España» en Mateu, J.F. y Nieto, M. (eds): Retorno al paisaje. El saber filosófico, cultural y científico del paisaje en España, EVREN, Valencia, pp. 389-474.

SIEFERLE, R. P. (2001): «Qué es la historia ecológica» en González de Molina, M. y Martínez Alier, J. (coords.): Naturaleza transformada. Icaria, Barcelona, pp. 31-52.

SWETNAM, T. W.; ALLEN, C.D.; BETANCOURT, J.L. (1999): «Applied historical ecology: using the past to manage for the future», Ecological Applications, $n^{\circ}$ 9(4), pp. 1189-1206.

TELLO, E. (coord.) (2006): «La transformación del territorio, antes y después de 1950: un lugar de encuentro transdisciplinar para el estudio del paisaje», Áreas, no 25,126 pp. (número monográfico). 
TERRADAS, J. (2003): «El paisaje y la ecología del paisaje», en Folch, R. (coord.): El territorio como sistema. Conceptos y métodos de ordenación. Diputació de Barcelona, Barcelona, pp. 57-72.

TURNER, M.G. (2005): «Ladscape ecology: Wath is the state of the science?», Annual Review of Ecology, Evolution and Systematic, $\mathrm{n}^{\circ} 36$, pp. 319-344.

UTRILLA, J.F.; LALIENA, C.; NAVARRO, G. (2003): «La evolución histórica del paisaje rural en los Pirineos durante la Edad Media: explotación agropecuaria y recursos forestales» en IRIARTE, I. y SABIO, A. (eds.) (2003): La construcción histórica del paisaje agrario en España y Cuba. Los Libros de la Catarata, Madrid, pp. 53-66.

VILA. J.; VARGA, D.; LLAUSÀS, A.; RIBAS, A. (2006): «Conceptos y métodos fundamentales en ecología del paisaje (landscape ecology). Una interpretación desde la geografía», Documents d'Anàlisi Geogràfica, no 48, pp. 151-166. 